\title{
Explicit Commutativity and Stability for the Heun's Linear Time-Varying Differential Systems
}

\author{
Salisu Ibrahim ${ }^{1}$ \\ ${ }^{1}$ Ishik University Erbil
}

July 7,2021

\begin{abstract}
This paper studies the commutativity and stability for the Heun's linear time-varying system (LTVS) with both zero and non-zero initial conditions(ICs). Given a LTVS A of order 2 , we find it's commutative pair, that is a new LTVS B of order $\mathrm{m}$ [?] n . Explicit commutative theories and conditions for second-order LTVSs are derived and solved to simplify and guarantee the equivalency between the connected input-output of systems A B and B A. The explicit results obtained are juxtaposed by simulation in order to investigate the commutativity of Heun's differential system, sensitivity of Heun's system, effects due to disturbance on Heun's system, robustness on Heun's system and problems regarding the stability of Heun's system. This findings will help to fill the gap on stability problem, system behaviors, commutativity theory, and general theory for solutions of differential equations, which has significant contribution to science and unlimited application in engineering, our results are verify using Heun's differential system as well as authenticated by Wolfrom Mathematica 11 and Matlab.
\end{abstract}

\section{Hosted file}

he.pdf available at https://authorea.com/users/424331/articles/529431-explicit-commutativityand-stability-for-the-heun-s-linear-time-varying-differential-systems

\section{Hosted file}

he.tex available at https://authorea.com/users/424331/articles/529431-explicit-commutativityand-stability-for-the-heun-s-linear-time-varying-differential-systems 
figures/ABO/AB0-eps-converted-to.pdf 
figures/AB2/AB2-eps-converted-to.pdf 
figures/AB3/AB3-eps-converted-to.pdf 
figures/b1/b1-eps-converted-to.pdf 
figures/b2/b2-eps-converted-to.pdf 
figures/b3/b3-eps-converted-to.pdf 
figures/d1/d1-eps-converted-to.pdf 
figures/d2/d2-eps-converted-to.pdf 
figures/d4/d4-eps-converted-to.pdf

a)

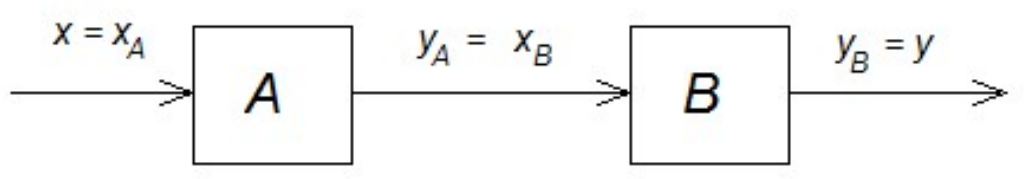

b)

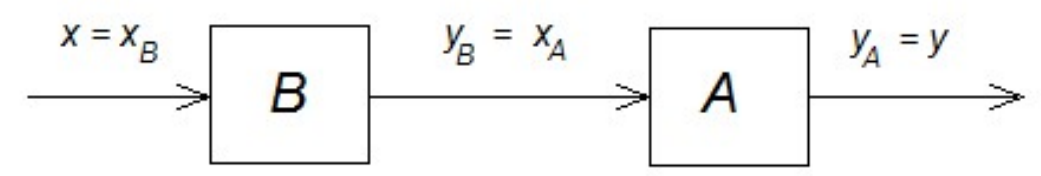

